\section{Bigram versatility and bigram frequency}

\author{
GENE E. TOPPER, WILLIAM H. MACEY \\ and ROBERT L. SOLSO* \\ Loyola University of Chicago, Chicago, Illinois 60626
}

It has been widely demonstrated that anagram solution time may be affected by the frequency of the bigrams of the solution word. Recently (cf. Solso, Topper, \& Macey, in press), bigram versatility, defined as the number of different words in which a given bigram appears, has been demonstrated also to play a significant role in anagram solution times. These two variables are seen as affecting total solution time in a nonindependent fashion, in that bigram frequency determines the set of words $S$ searches for in memory and bigram versatility determines the size of the set of words through which $S$ must search.

The present paper reports a data base from which solution anagrams may be constructed on the basis of both bigram frequency and bigram versatility. Table 1 presents the versatility and frequency counts of the bigrams AA through $\mathrm{ZZ}$. Bigram versatilities were calculated from a $10 \%$ randomly selected group of words from a stratified sample of 10 successive items of the words throughout the Thorndike-Lorge (1944) word list of frequency one per million and over. Every bigram in the word used was recorded for its occurrence. Corresponding frequencies for the bigrams were selected from Underwood \& Schulz (1960).

Table 1

Versatility and Frequency Counts of Bigrams (B) AA-MY*

\begin{tabular}{lrrlrr}
\hline B & V & F & B & V & \multicolumn{1}{c}{ F } \\
\hline AA & 0 & 3 & AN & 181 & 3182 \\
AB & 54 & 639 & AO & 0 & 1 \\
AC & 60 & 1218 & AP & 38 & 703 \\
AD & 46 & 747 & AQ & 0 & 1 \\
AE & 1 & 6 & AR & 139 & 2389 \\
AF & 12 & 175 & AS & 61 & 1540 \\
AG & 36 & 588 & AT & 164 & 2363 \\
AH & 2 & 25 & AU & 22 & 362 \\
AI & 49 & 829 & AV & 20 & 479 \\
AJ & 1 & 147 & AW & 11 & 110 \\
AK & 18 & 439 & AX & 4 & 62 \\
AL & 152 & 1690 & AY & 17 & 198 \\
AM & 44 & 759 & AZ & 5 & 30
\end{tabular}

$* V=$ versatility, $\underline{N} V=12,344$ bigrams; $F=$ frequency (Underwood \& Schulz, 1960), $N F=1,000$ words from $T \cdot L$ plus 15,000 words running text. *Requests for reprints should be sent to Robert L. Solso,
Loyola University of Chicago, 6525 North Sheridan Road, Chicago, Illinois 60626.

\begin{tabular}{|c|c|c|c|c|c|}
\hline B & $\mathrm{V}$ & F & B & $\mathbf{V}$ & $\mathbf{F}$ \\
\hline BA & 31 & 413 & EP & 23 & 320 \\
\hline BB & 5 & 20 & EQ & 10 & 159 \\
\hline BD & 2 & 1 & $\mathbf{E R}$ & 264 & 4034 \\
\hline $\mathrm{BE}$ & 45 & 761 & ES & 129 & 2222 \\
\hline BI & 21 & 234 & ET & 71 & 1268 \\
\hline BJ & 0 & 7 & EU & 7 & 32 \\
\hline BK & 0 & 1 & EV & 28 & 377 \\
\hline BL & 62 & 747 & EW & 15 & 312 \\
\hline BN & 1 & 1 & EX & 21 & 603 \\
\hline BO & 31 & 404 & EY & 16 & 153 \\
\hline BR & 31 & 197 & EZ & 0 & 35 \\
\hline BS & 9 & 58 & FA & 21 & 420 \\
\hline BT & 1 & 2 & FB & 0 & 2 \\
\hline $\mathrm{BU}$ & 20 & 522 & $\mathrm{FE}$ & 22 & 712 \\
\hline BV & 1 & 2 & $\mathrm{FF}$ & 18 & 328 \\
\hline BW & 0 & 5 & FI & 37 & 721 \\
\hline BY & 2 & 68 & FL & 17 & 148 \\
\hline $\mathrm{CA}$ & 88 & 1055 & FN & 0 & 3 \\
\hline $\mathrm{CC}$ & 9 & 225 & FO & 33 & 710 \\
\hline $\mathrm{CE}$ & 68 & 1596 & FR & 22 & 303 \\
\hline CG & 0 & 2 & FS & 0 & 6 \\
\hline $\mathrm{CH}$ & 64 & 1297 & FT & 9 & 200 \\
\hline $\mathrm{CI}$ & 47 & 603 & FU & 27 & 349 \\
\hline CK & 36 & 676 & FY & 9 & 92 \\
\hline $\mathrm{CL}$ & 24 & 648 & GA & 31 & 398 \\
\hline $\mathrm{CO}$ & 109 & 1117 & GB & 0 & 1 \\
\hline $\mathrm{CQ}$ & 2 & 1 & GD & 1 & 7 \\
\hline $\mathrm{CR}$ & 36 & 432 & $\mathrm{GE}$ & 51 & 883 \\
\hline CS & 0 & 5 & GF & 2 & 3 \\
\hline $\mathrm{CT}$ & 46 & 607 & GG & 10 & 101 \\
\hline CU & 29 & 459 & GH & 21 & 684 \\
\hline $\mathrm{CY}$ & 15 & 77 & GI & 18 & 456 \\
\hline DA & 28 & 342 & GL & 18 & 192 \\
\hline $\mathrm{DB}$ & 1 & 1 & GM & 1 & 59 \\
\hline DD & 11 & 75 & GN & 17 & 47 \\
\hline $\mathrm{DE}$ & 119 & 1476 & GO & 23 & 164 \\
\hline DF & 3 & 58 & GP & 0 & 5 \\
\hline DG & 5 & 98 & GR & 38 & 490 \\
\hline DH & 2 & 5 & GS & 3 & 64 \\
\hline DI & 82 & 706 & GT & 2 & 112 \\
\hline DK & 0 & 35 & GU & 17 & 127 \\
\hline DL & 16 & 140 & GY & 10 & 59 \\
\hline DM & 6 & 99 & HA & 55 & 1396 \\
\hline DN & 4 & 56 & HB & 0 & 5 \\
\hline DO & 29 & 397 & HD & 1 & 2 \\
\hline DP & 2 & 2 & $\mathrm{HE}$ & 75 & 2897 \\
\hline DR & 15 & 472 & $\mathrm{HF}$ & 4 & 4 \\
\hline DS & 8 & 95 & HG & 0 & 1 \\
\hline DT & 6 & 1 & HI & 54 & 1003 \\
\hline DU & 20 & 165 & $\mathrm{HL}$ & 5 & 18 \\
\hline DV & 4 & 83 & HM & 3 & 50 \\
\hline DW & 1 & 29 & HN & 6 & 11 \\
\hline DY & 9 & 152 & $\mathrm{HO}$ & 54 & 1129 \\
\hline EA & 80 & 2516 & HP & 0 & 1 \\
\hline EB & 9 & 26 & HR & 10 & 193 \\
\hline EC & 54 & 729 & HS & 0 & 12 \\
\hline ED & 88 & 1176 & HT & 17 & 496 \\
\hline $\mathrm{EE}$ & 40 & 1046 & $\mathrm{HU}$ & 19 & 478 \\
\hline $\mathrm{EF}$ & 25 & 201 & HV & 0 & 1 \\
\hline $\mathrm{EG}$ & 21 & 295 & $\mathrm{HW}$ & 0 & 6 \\
\hline EH & 4 & 54 & HY & 8 & 46 \\
\hline EI & 10 & 397 & IA & 61 & 421 \\
\hline EJ & 1 & 1 & IB & 17 & 80 \\
\hline EK & 5 & 70 & IC & 105 & 1309 \\
\hline EL & 100 & 1579 & ID & 48 & 495 \\
\hline EM & 40 & 691 & IE & 40 & 670 \\
\hline EN & 180 & 3203 & $\mathrm{IF}$ & 17 & 513 \\
\hline EO & 12 & 89 & IG & 46 & 925 \\
\hline
\end{tabular}


Table 1 Continued

Table 1 Continued

\begin{tabular}{|c|c|c|c|c|c|c|c|c|c|c|c|}
\hline B & $\mathrm{V}$ & $\mathbf{F}$ & B & $\mathbf{V}$ & $\mathrm{F}$ & B & $\mathbf{V}$ & $F$ & $\mathrm{~B}$ & $\mathbf{V}$ & $\mathrm{F}$ \\
\hline IK & 3 & 49 & MO & 45 & 852 & $\mathbf{P M}$ & 1 & 12 & $\mathrm{TN}$ & 4 & 16 \\
\hline IL & 67 & 1083 & MP & 38 & 382 & PN & 0 & 1 & TO & 63 & 1213 \\
\hline IM & 44 & 441 & MR & 0 & 11 & PO & 49 & 818 & TP & 0 & 1 \\
\hline IN & 229 & 3902 & MS & 8 & 49 & PP & 14 & 451 & TR & 66 & 1028 \\
\hline IO & 126 & 1615 & MT & 1 & 1 & PR & 65 & 840 & TS & 8 & 342 \\
\hline IP & 23 & 234 & MU & 14 & 148 & PS & 4 & 32 & $\mathrm{TT}$ & 32 & 418 \\
\hline IQ & 5 & 13 & MY & 6 & 77 & PT & 13 & 283 & $\mathrm{TU}$ & 39 & 472 \\
\hline IR & 37 & 1061 & NA & 65 & 637 & PU & 50 & 220 & TW & 3 & 65 \\
\hline IS & 105 & 2051 & NB & 3 & 16 & PY & 3 & 72 & TY & 48 & 725 \\
\hline IT & 114 & 1334 & $\mathrm{NC}$ & 62 & 795 & QU & 31 & 430 & $\mathrm{TZ}$ & 0 & 4 \\
\hline IU & 6 & 11 & ND & 77 & 1650 & $\mathbf{R A}$ & 140 & 1488 & UA & 19 & 171 \\
\hline IV & 46 & 594 & $\mathrm{NE}$ & 103 & 1689 & RB & 9 & 70 & UB & 15 & 90 \\
\hline IW & 0 & 1 & NF & 19 & 124 & $\mathrm{RC}$ & 13 & 515 & UC & 23 & 205 \\
\hline IX & 4 & 124 & NG & 91 & 1998 & RD & 31 & 354 & UD & 15 & 139 \\
\hline IZ & 14 & 50 & NH & 5 & 18 & RE & 210 & 3791 & $\mathrm{UE}$ & 17 & 320 \\
\hline $\mathbf{J A}$ & 5 & 83 & NI & 60 & 574 & RF & 6 & 126 & UF & 6 & 171 \\
\hline JE & 6 & 93 & NJ & 2 & 8 & RG & 15 & 165 & UG & 15 & 249 \\
\hline JI & 2 & 2 & NK & 13 & 142 & RH & 1 & 9 & UI & 24 & 244 \\
\hline JO & 6 & 204 & $\mathrm{NL}$ & 9 & 73 & RI & 134 & 1515 & UK & 1 & 3 \\
\hline $\mathrm{JU}$ & 8 & 163 & $\mathbf{N M}$ & 4 & 25 & RK & 7 & 250 & UL & 57 & 817 \\
\hline KA & 6 & 51 & $\mathrm{NN}$ & 17 & 235 & RL & 19 & 277 & $\mathrm{UM}$ & 30 & 288 \\
\hline KB & 2 & 7 & NO & 32 & 728 & $\mathrm{RM}$ & 18 & 365 & UN & 79 & 1147 \\
\hline KD & 0 & 2 & NP & 2 & 3 & $\mathbf{R N}$ & 23 & 282 & UO & 5 & 37 \\
\hline $\mathrm{KE}$ & 44 & 828 & NQ & 3 & 69 & RO & 97 & 1721 & UP & 18 & 311 \\
\hline $\mathrm{KF}$ & 0 & 6 & NR & 3 & 26 & $\mathbf{R P}$ & 12 & 28 & UR & 75 & 1304 \\
\hline KG & 0 & 1 & NS & 55 & 466 & RR & 26 & 217 & US & 90 & 1079 \\
\hline KH & 0 & 3 & NT & 158 & 2852 & RS & 31 & 470 & UT & 34 & 1137 \\
\hline $\mathrm{KI}$ & 12 & 205 & NU & 13 & 269 & RT & 57 & 687 & UV & 0 & 6 \\
\hline KL & 6 & 34 & NV & 7 & 155 & $\mathrm{RU}$ & 30 & 140 & UX & 3 & 10 \\
\hline $\mathrm{KN}$ & 6 & 88 & NW & 2 & 13 & RV & 10 & 137 & UY & 0 & 6 \\
\hline $\mathrm{KO}$ & 2 & 13 & $\mathrm{NX}$ & 1 & 3 & RW & 3 & 51 & $\mathrm{UZ}$ & 0 & 7 \\
\hline KP & 0 & 1 & NY & 11 & 85 & RX & 0 & 2 & VA & 26 & 379 \\
\hline KR & 1 & 9 & $\mathrm{NZ}$ & 1 & 5 & RY & 35 & 459 & $\mathrm{VE}$ & 104 & 2040 \\
\hline KS & 2 & 55 & $\mathbf{O A}$ & 17 & 66 & SA & 37 & 566 & VI & 36 & 613 \\
\hline $\mathrm{KT}$ & 0 & 1 & $\mathrm{OB}$ & 19 & 121 & SB & 1 & 21 & Vo & 10 & 122 \\
\hline KU & 0 & 5 & $O C$ & 22 & 578 & $\mathrm{SC}$ & 36 & 329 & VR & 0 & 1 \\
\hline KY & 2 & 34 & OD & 25 & 394 & SD & 1 & 1 & VU & 1 & 1 \\
\hline $\mathrm{LA}$ & 89 & 1307 & $\mathrm{OE}$ & 5 & 93 & SE & 90 & 1865 & VY & 0 & 12 \\
\hline LB & 2 & 14 & OF & 8 & 737 & SF & 2 & 20 & WA & 27 & 723 \\
\hline LC & 5 & 55 & OG & 22 & 217 & $\mathrm{SG}$ & 1 & 49 & WB & 2 & 6 \\
\hline LD & 22 & 682 & $\mathrm{OH}$ & 7 & 6 & SH & 59 & 1203 & WC & 0 & 2 \\
\hline LE & 168 & 2794 & OI & 14 & 113 & SI & 76 & 1057 & WD & 2 & 117 \\
\hline LF & 7 & 75 & $\mathrm{OJ}$ & 0 & 3 & SK & 9 & 111 & $\mathrm{WE}$ & 15 & 779 \\
\hline LG & 2 & 17 & OK & 9 & 406 & SL & 20 & 349 & WH & 14 & 700 \\
\hline LH & 1 & 1 & $\mathrm{OL}$ & 64 & 950 & SM & 17 & 90 & WI & 18 & 338 \\
\hline LI & 99 & 1271 & $\mathrm{OM}$ & 45 & 740 & SN & 7 & 47 & WK & 1 & 4 \\
\hline LK & 5 & 24 & ON & 220 & 3107 & So & 43 & 920 & WL & 6 & 17 \\
\hline LL & 82 & 1298 & 00 & 46 & 716 & SP & 45 & 707 & WM & 1 & 1 \\
\hline LM & 7 & 181 & OP & 24 & 268 & SQ & 3 & 12 & WN & 11 & 212 \\
\hline LN & 0 & 9 & $\mathrm{OQ}$ & 0 & 1 & SR & 0 & 1 & WO & 16 & 288 \\
\hline LO & 64 & 1054 & OR & 149 & 2260 & SS & 83 & 1037 & $\mathrm{WP}$ & 2 & 1 \\
\hline $\mathrm{LP}$ & 8 & 175 & OS & 40 & 592 & ST & 136 & 3473 & WR & 5 & 64 \\
\hline LR & 2 & 6 & OT & 37 & 738 & SU & 40 & 598 & WS & 0 & 23 \\
\hline LS & 10 & 227 & OU & 92 & 2118 & SW & 7 & 100 & WT & 1 & 6 \\
\hline LT & 14 & 140 & OV & 23 & 766 & SY & 9 & 180 & $W Y$ & 2 & 16 \\
\hline $\mathbf{L U}$ & 27 & 290 & OW & 36 & 676 & $\mathrm{TA}$ & 88 & 913 & $\mathbf{X A}$ & 4 & 102 \\
\hline LV & 6 & 127 & OX & 4 & 38 & $\mathrm{~TB}$ & 1 & 5 & $\mathrm{XC}$ & 4 & 62 \\
\hline LW & 0 & 13 & OY & 1 & 111 & $\mathrm{TC}$ & 10 & 163 & $\mathrm{XE}$ & 2 & 71 \\
\hline LY & 105 & 1100 & $\mathrm{OZ}$ & 1 & 5 & TD & 0 & 2 & $X F$ & 1 & 17 \\
\hline MA & 84 & 1210 & PA & 48 & 1098 & $\mathrm{TE}$ & 174 & 2899 & $\mathrm{XH}$ & 0 & 3 \\
\hline MB & 20 & 288 & PB & 1 & 4 & $\mathrm{TF}$ & 5 & 19 & XI & 6 & 120 \\
\hline ME & 72 & 1161 & PE & 83 & 1263 & TG & 1 & 3 & $\mathrm{XL}$ & 1 & 10 \\
\hline MF & 0 & 5 & PF. & 0 & 14 & $\mathrm{TH}$ & 71 & 2879 & $\mathrm{XM}$ & 0 & 2 \\
\hline MI & 48 & 720 & PH & 29 & 157 & $\mathrm{TI}$ & 189 & 2497 & XO & 1 & 1 \\
\hline ML & 2 & 4 & PI & 30 & 281 & TJ & 0 & 1 & $\mathbf{X P}$ & 7 & 237 \\
\hline MM & 18 & 52 & PK & 0 & 1 & $T L$ & 20 & 639 & $\mathrm{XT}$ & 5 & 125 \\
\hline MN & 7 & 4 & PL & 30 & 498 & $\mathrm{TM}$ & 12 & 63 & $X U$ & 1 & 15 \\
\hline
\end{tabular}


Table 1 Continued

\begin{tabular}{lrrlrr}
\hline B & V & F & B & V & F \\
\hline YA & 1 & 96 & YR & 8 & 23 \\
YB & 0 & 6 & YS & 10 & 56 \\
YC & 4 & 12 & YT & 3 & 17 \\
YD & 2 & 19 & YV & 0 & 1 \\
YE & 5 & 105 & YW & 1 & 19 \\
YG & 2 & 8 & YX & 0 & 4 \\
YH & 0 & 2 & ZA & 3 & 10 \\
YI & 3 & 64 & ZE & 14 & 88 \\
YL & 2 & 84 & ZI & 1 & 10 \\
YM & 3 & 33 & ZL & 1 & 4 \\
YN & 2 & 10 & ZO & 3 & 8 \\
YO & 6 & 251 & ZY & 2 & 7 \\
YP & 5 & 34 & ZZ & 2 & 9 \\
& & & & &
\end{tabular}

\section{REFERENCES}

Solso, R. L., Topper, G. E., M Macey, W. H. Anagram solution as a function of bigram versatility. Journal of Experimental Psychology, in press.

Thorndike E. L., \& Lorge, I. The teacher's word book of 30,000 words. New York: Bureau of Publications, Teachers College, Columbia University, 1944.

Underwood, B. J., \& Schulz, R. W. Meaningfulness and verbal learning. Chicago: Lippincott, 1960.

(Received for publication October 5, 1972; accepted October 27, 1972.)

\section{An on-off switch}

for solid state programming systems*

\section{PAUL LEWIS \\ Ohio University, Athens, Ohio 45701}

One difficulty with some solid state programming systems is that modules (e.g., counters, lamp drivers, etc.) requiring both 28 - and $12-\mathrm{V}$ power must always have the $12 \mathrm{~V}$ applied before the $28 \mathrm{~V}$. If $28 \mathrm{~V}$ is on without the 12 , serious damage can occur to logic drivers and driven equipment, such as counters, since no bias exists to control the $28 \mathrm{~V}$.

Figure 1 describes an inexpensive (approximately \$22) circuit which handles the problem and allows enough current to control large programming systems. Two buttons are employed. One button provides power (both 12 and $28 \mathrm{~V}$ ) to the programming modules, the other turns the power off. Pushing the "on" button supplies $12 \mathrm{~V}$ first, then the $28 \mathrm{~V}$. The "off" button turns $28 \mathrm{~V}$ off, and a few milliseconds later the $12 \mathrm{~V}$ disengages.

ON CYCLE: When the "on" pushbutton (S1) is depressed, Silicon-Controlled Rectifier SCR 1. is gated to an "on" state. Consequently, Relay RY1 is energized, thus connecting the bias supply to the equipment. Also, the timing cycle on the unijunction transistor $(\mathrm{Q} 1)$ is initiated. After a predetermined interval (R4, C2), the unijunction (Q1) gates Silicon-Controlled Rectifier

*Work on this note was supported by NIMH Grant IR01 MH9593-1 to Ohio University. The author thanks Dennis Coolidge and Craig Pelton for their technical assistance in the design and construction of this switch. Reprints may be obtained from Paul Lewis, Psychology Department, Porter Hall, Ohio University, Athens, Ohio 45701 .

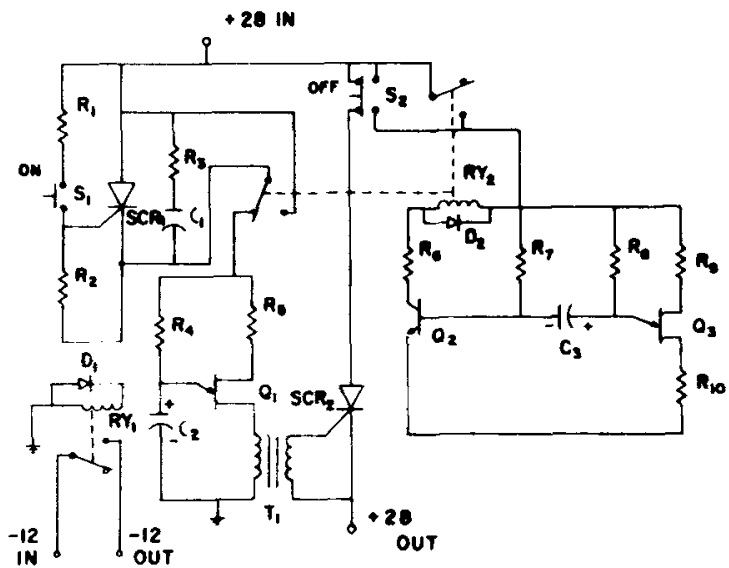

Fig. 1. Circuit diagram for on-off switch to be used with solid state programming systems.

Table 1

Parts List

\begin{tabular}{ll}
$\mathrm{C}_{1}$ & $.47 \mu \mathrm{F}$ \\
$\mathrm{C}_{2}, \mathrm{C}_{3}$ & $10 \mu \mathrm{F}$ (see text) \\
$\mathrm{D}_{1}, \mathrm{D}_{2}$ & $200 \mathrm{PIV}, 1 \mathrm{~A}$ \\
$\mathrm{Q}_{1}, \mathrm{Q}_{3}$ & $2 \mathrm{~N} 2646$ (unijunction) \\
$\mathrm{Q}_{2}$ & $2 \mathrm{~N} 1302$ (bipolar) \\
$\mathrm{R}_{1}$ & $620 \Omega$ \\
$\mathrm{R}_{2}$ & $1 \mathrm{~K}$ \\
$\mathrm{R}_{3}$ & $2 \Omega$ \\
$\mathrm{R}_{4}, \mathrm{R}_{8}$ & $39 \mathrm{k}$ (see text) \\
$\mathrm{R}_{5}, \mathrm{R}_{9}$ & $330 \Omega$ \\
$\mathrm{R}_{6}$ & $200 \Omega$ \\
$\mathrm{R}_{7}$ & $6.8 \mathrm{k}$ \\
$\mathrm{R}_{1}$ & $10 \Omega$ \\
$\mathrm{RY}_{1}$ & $24-\mathrm{V}$ coil SPST 10A contacts \\
$\mathrm{RY}_{2}$ & $24-\mathrm{V}$ coil DPDT 2A contacts \\
$\mathrm{S}_{1}$ & SPST momentary action switch 24 V, 2A \\
$\mathrm{S}_{2}$ & SPDT momentary action switch 24 V, 2A \\
$\mathrm{SCR}_{1}$ & $\mathrm{RCA}$ C106B \\
$\mathrm{SCR}_{2}$ & $\mathrm{RCA} \mathrm{C} 122 \mathrm{D}$ \\
$\mathrm{T}_{1}$ & Pulse transformer 1:1 ratio \\
\hline
\end{tabular}

SCR2, which supplies power to the $28-\mathrm{V}$ circuits.

OFF CYCLE: Depressing the "off" pushbutton (S2) initiates two simultaneous functions. First, the $28-\mathrm{V}$ supply is disengaged. Second, the dropout relay timer is energized, which (1) maintains bias to the dropout circuit, (2) short circuits Silicon-Controlled Rectifier SCR1, and (3) disconnects the "on" timing circuit. All above functions are controlled by Relay RY2. The dropout timer, consisting of Transistor Q2 and Unijunction Q3, interval is determined by Resistor R3 and Capacitor $\mathrm{C} 3$. At the end of the predetermined timing interval, Transistor Q2 is switched to an "off" state, which in turn releases Relay RY2. This, in turn, releases Relay RY1, turning "off" the $12-\mathrm{V}$ bias.

Diodes D1 and D2 suppress transient voltages (i.e., $\mathrm{L}$ di/dt). Also, Resistor R3 and Capacitor C1 insure voltage turn off on Silicon-Controlled Rectifier SCR 1 (i.e., $d v / d t$ ).

Three circuits have been in use for 2 months without a failure.

Parts are listed in Table 1.

(Received for publication October 27, 1972; accepted November 12,1972 .) 